\title{
Urinary stone detection and characterisation with dual-energy CT urography after furosemide intravenous injection: preliminary results
}

\author{
Diomidis Botsikas • Catrina Hansen • \\ Salvatore Stefanelli • Christoph D. Becker • \\ Xavier Montet
}

Received: 1 July 2013 /Revised: 30 August 2013 / Accepted: 15 September 2013 / Published online: 2 October 2013

(C) European Society of Radiology 2013

\begin{abstract}
Objectives To investigate the added advantage of IV furosemide injection and the subsequent urine dilution in the detection of urinary calculi in the excretory phase of dual-source dual-energy (DE) computed tomography (CT) urography, and to investigate the feasibility of characterising the calculi through diluted urine. Methods Twenty-three urinary calculi were detected in 116 patients who underwent DECT urography for macroscopic haematuria with a split bolus two- or three-acquisition protocol, including a true unenhanced series and at least a mixed nephrographic excretory phase. Virtual unenhanced images were reconstructed from contrast-enhanced DE data. Calculi were recorded on all series and characterised based on their Xray absorption characteristics at $100 \mathrm{kVp}$ and $140 \mathrm{kVp}$ in both true unenhanced and nephrographic excretory phase series.

Results All calculi with a diameter more than $2 \mathrm{~mm}$ were detected in the virtual unenhanced phase and in the nephrographic excretory phase. Thirteen of these calculi could be characterised in the true unenhanced phase and in the mixed nephrographic excretory phase. The results were strictly identical for both phases, six of them being recognised as non-uric acid calculi and seven as uric acid calculi.

Conclusions Mixed nephrographic excretory phase DECT after furosemide administration allows both detection and characterisation of clinically significant calculi, through the diluted urine. Key points

- Urinary tract stones can be detected on excretory phase through diluted urine.

- Urinary tract stone characterisation with dual-energy CT (DECT) is possible through diluted urine.
\end{abstract}

D. Botsikas $(\triangle) \cdot$ C. Hansen $\cdot$ S. Stefanelli $\cdot$ C. D. Becker $\cdot$ X. Montet

Radiology Department, Geneva University Hospital, Rue

Gabrielle-Perret-Gentil 4, 1205 Geneva, Switzerland

e-mail: diomidis.botsikas@hcuge.ch
- A dual energy split-bolus CT urography simultaneously enables urinary stone detection and characterisation.

Keywords Dual-energy CT (DECT) urography · Split-bolus CT urography protocol $\cdot$ Furosemide $\cdot$ Urinary stones composition $\cdot$ Urinary stones detection

\section{Introduction}

Computed tomography (CT) urography is the diagnostic method of choice for the investigation of haematuria [1]. Urinary tract calculi are the most frequent cause of haematuria, urinary tract infection excluded, in patients less than 40 years old, with no risk factors for malignancy. For the detection of calculi in this patient group, an unenhanced low-dose CT of kidneys, ureters and bladder is the imaging method of choice. CT urography is significantly inferior to unenhanced CT for the detection of urinary calculi [1]. In order to decide which patient should undergo which imaging examination, the radiologist often faces a dilemma and, frequently, even if calculi are detected on the unenhanced CT, the work schedule will continue, with one or more acquisitions after intravenous iodinated contrast material injection, for the detection of an eventual tumoural lesion. If all clinically important information concerning urinary calculi could be acquired from one enhanced series only, the unenhanced acquisition could be skipped.

Dual-source dual-energy (DE) CT has the ability to create virtual unenhanced images on the basis of data from the enhanced acquisition. However, in the late excretory phase of CT urography, urine in the collecting system is densely opacified. Virtual unenhanced images of the late excretory phase are of suboptimal quality because of the densely opacified urine. For this reason, virtual unenhanced imaging from the excretory phase acquisition has been shown to be of moderate accuracy in the detection of urinary calculi [2]. 
Dual-source DECT has been shown to accurately characterise calculi according to their chemical composition, based on their absorption characteristics of X-rays at different $\mathrm{kVp}$ settings on unenhanced studies [3-5].

In the excretory phase of a standard CT urography, calculi are surrounded by urine with similar densities. Because of this, and because of a partial volume effect at the periphery of the calculus, characterisation is impossible. Densely opacified urine has been shown in vitro to pose an obstacle to characterising the calculi, especially the smallest ones [6].

Intravenous (IV) furosemide administration, before the acquisition of excretory phase CT urography, results in a lower contrast density of the opacified urine in the collecting system.

The purpose of this study was to investigate the added advantage of IV furosemide injection and the subsequent urine dilution, in the detection of urinary calculi in the excretory phase of dual-source DECT urography and to investigate the feasibility of characterising the calculi through diluted urine.

\section{Materials and methods}

\section{Patient population}

The study was approved by the ethics committee of our hospital.

The DECT urographies of 120 consecutive adult patients performed in our hospital from September 2011 to August 2012 were reviewed retrospectively.

The indications for the examination were: macroscopic haematuria (43), microscopic haematuria (1), investigation of known or suspected renal tumour (15), suspicion, followup or extension control of urothelial cancer (41), morphological control in the case of persistent urinary tract infections or obstructive semiology (12), investigation of known or clinical suspicion of urinary tract calculi (4) and other (4).

CT urography is not performed in our hospital in the following patients: pregnant women, patients aged below 18 years, and those with acute renal colic. Furosemide is not injected into patients with hypotension and known allergy to sulphonamides.

Four patients in total were excluded: three of them because of significant artefacts generated from bilateral hip prostheses and one because of utilisation of a different protocol by the referring radiographer. The remaining 116 patients formed the population of the study ( 92 men and 24 women; mean age $70.22 \pm 14.27$ years; age range 19-99). The mean bodyweight of the patients was $75.22 \pm 14.13 \mathrm{~kg}$. The patients' mean BMI was $26.2 \pm 4.61$.

Imaging protocol

DECT urography was performed on a second generation dualsource DECT system (SOMATOM Definition Flash; Siemens
Healthcare, Forchheim, Germany), using an unenhanced and one- or two-phase enhanced acquisitions protocol, according to the clinical setting. The examination protocol was as follows. Patients emptied their bladder just before the examination. First, unenhanced DECT acquisitions of the abdomen and pelvis were obtained. All patients were subsequently administered $20 \mathrm{mg}$ furosemide (Lasix; Sanofi Aventis, Laval, Quebec, Canada) intravenously and $1 \mathrm{~min}$ later a dose of $1.2 \mathrm{ml} / \mathrm{kg}$ Iohexol (Accupaque 350; GE Healthcare, Milwaukee, WI, USA) intravenously at a rate of $2 \mathrm{ml} / \mathrm{s}$. For the threeacquisition protocol, a late arterial corticomedullary phase acquisition of the abdomen and pelvis was obtained $35 \mathrm{~s}$ after the beginning of the contrast medium injection. A second injection of $0.8 \mathrm{ml} / \mathrm{kg}$ of the same contrast agent at the same rate followed 7 min after the first injection and a mixed nephrographic excretory imaged series was obtained $100 \mathrm{~s}$ after the beginning of the second contrast bolus injection, for both protocols.

In total, six patients underwent imaging with the twoacquisitions and 110 with the three-acquisition protocol.

The acquisition parameters for all phases were: $\mathrm{DE}$ mode with one tube at $100 \mathrm{kVp}$ and the other at $140 \mathrm{kVp}$, current modulation (4D care dose ${ }^{\circledR}$ ) with reference $250 \mathrm{mAs}$ for the $100 \mathrm{kVp}$ tube, collimation of $32 \times 2 \times 0.625 \mathrm{~mm}$, gantry rotation time $0.5 \mathrm{~s}$ and a pitch of 0.6. All three acquisitions were reconstructed as 2-mm-thick sections. Radiation dose was calculated for each acquisition separately and was expressed in $\mathrm{mSv}$. These values were estimated by multiplying the total dose-length product provided by the CT console for each acquisition, by a normalising coefficient of 0.015 .

Image analysis

All DECT urography examinations were transferred on an independent workstation (Syngo DE; Siemens Healthcare, Erlangen Germany) and evaluated on consensus by two radiologists (11 and 3 years of experience in genitourinary radiology), who were blinded for series technique, patients identities and the final diagnosis as established from the true unenhanced series that was used as a "gold standard".

\section{Calculus detection}

In the reading of DECT urography examinations the two readers evaluated in consensus the unenhanced series, the virtual unenhanced series issuing from the mixed nephrographic excretory acquisition and the mixed nephrographic excretory series without iodine subtraction for the presence of urinary tract calculi.

Virtual unenhanced images were obtained from the mixed nephrographic excretory DE acquisition by using the "liver VNC" algorithm of the SyngoDE software.

All series were evaluated on the axial plane with the help of any additional multiplanar or three-dimensional reconstructions if necessary. Window settings were freely changed according to 
the excreted contrast densities and consequently were different for each examination. When a calculus was found, its position was noted after dividing the urinary tract into seven anatomical segments: 1 , precalyceal or calyceal; 2 , renal pelvic; 3 , proximal ureteral; 4 , middle ureteral; 5 , distal ureteral; 6 , ureterovesical junction (UVJ); 7, intra-vesical.

The maximal diameters of the calculi were also noted, measured in the axial plane, with bone window settings (window level $300 \mathrm{HU}$; window width 1,500 HU).

\section{Characterisation of the calculi}

When a calculus was detected in either the unenhanced or the mixed nephrographic pyelographic phase, the data of the corresponding DE series were further reconstructed using the "kidney stone" algorithm of Syngo DE software.

This software attributed two different coloured overlays to different calculi, according to their chemical composition: red for uric acid and blue for non-uric acid. The material decomposition achieved by this software is based on the different absorption of X-rays of different energies.

Results of calculus characterisation on the basis of the mixed nephrographic excretory acquisition were compared with those of characterisation on the basis of the unenhanced DE acquisition.

\section{Statistical analysis}

Statistical analysis was performed with Prism (Prism, version 5d, 2010; GraphPad Software, San Diego, CA, USA) and Microsoft Excel (Excel for MAC version 14.3.4, 2011; Microsoft Corporation, Redmond, Washington, USA). For continuous values, the results are presented as mean \pm standard error. Normally distributed data sets, established by the Kolmogorov-Smirnov test, were compared using Student's $t$ test. Two-sided testing was used. Differences were considered significant at $P<0.05$.

\section{Results}

Of the 116 patients included in the study, ten patients had a total of 23 calculi detected on the unenhanced phase. The mean size of the calculi was $6.46 \pm 3.77 \mathrm{~mm}$ (range 1.3-13 mm). Twelve of these calculi were of precalyceal or calyceal localisation (mean size $3.89 \pm 2.11 \mathrm{~mm}$; range $1.3-7.3 \mathrm{~mm}$ ). Seven were located in the bladder (mean size 10.9 $\pm 2.02 \mathrm{~mm}$; range $7.3-13 \mathrm{~mm}$ ). Two were found in the renal pelvis and measure 4.9 and $3.5 \mathrm{~mm}$; one was localised on the UVJ $(8 \mathrm{~mm})$ and one in the distal ureter (7.5 mm).

Of these calculi, 18 were detected on the virtual unenhanced series; mean size $7.3 \pm 3.2 \mathrm{~mm}$; range $3.3-11.8 \mathrm{~mm}$ (seven calyceal, seven in the bladder, two in the renal pelvis, one in the distal ureter and one in the UVJ). Overall sensitivity was $78.2 \%$; for calculi $\geq 2 \mathrm{~mm}$, sensitivity was $100 \%$ (Table 1 ). The five calculi that were not detected on the virtual unenhanced series measured $\leq 2 \mathrm{~mm}$ and were of calyceal or precalyceal location.

In the mixed nephrographic excretory series, 20 calculi in total were identified with a mean size of $6.8 \pm 3.7 \mathrm{~mm}$ (nine calyceal, seven in the bladder, two in the renal pelvis, one in the distal ureter and one in the UVJ). Overall sensitivity was $86.9 \%$; for calculi $\geq 2 \mathrm{~mm}$, sensitivity was $100 \%$ (Table 1 ). The three calculi missed by this series measured $\leq 2 \mathrm{~mm}$ and were of calyceal or precalyceal location.

Thirteen of the calculi could be characterised in the true unenhanced and the nephrographic excretory phase and the results were strictly identical for both series, six of them being identified as non-uric acid calculi (Fig. 1) and seven as uric acid calculi (Fig. 2). The mean size of these 13 calculi was $8.49 \pm 3.07 \mathrm{~mm}$; range $3.5-13 \mathrm{~mm}$ and was significantly higher than the size of the calculi that could not be characterised by DECT $(2.91 \pm 1.58$; range $1.3-6 \mathrm{~mm} ; P=0.000017$ ).

Mean radiation doses were $9.077 \pm 2.917,9.468 \pm 3.045$ and $9.326 \pm 2.902 \mathrm{mSv}$ respectively for unenhanced, corticomedullary and mixed nephrographic excretory phases.

\section{Discussion}

Detection of urinary calculi in opacified urine by using DECT has been previously reported, with suboptimal results; overall sensitivity for detecting calculi was $63 \%$ ( 27 out of 43 calculi) per calculus [7]. In another study, sensitivity of the virtual unenhanced series for calculi was found to be moderate, even for calculi larger than $2.9 \mathrm{~mm}$ (76\%) [8]. This was probably due to dense contrast-enhanced urine surrounding calculi and creating a partial volume effect, resulting in oversubtraction and thus elimination of calculi, or insufficient subtraction of iodine. Karlo et al. [9], in a recent study, reported better results for the virtual unenhanced series reconstructed from the mixed nephrographic excretory phase. In this study, $83 \%$ of calculi were detected. Missed calculi had a mean size of $2.5 \mathrm{~mm}$ (range 1-4 mm) and were significantly smaller than the calculi correctly identified. In our study, the missed calculi were all

Table 1 Overall sensitivities and sensitivities for calculi greater than $2 \mathrm{~mm}$ for the unsubtracted mixed nephrographic excretory phase and the corresponding virtual unenhanced image series

Sensitivities of different series for stone detection

\begin{tabular}{lclc}
\hline \multirow{2}{*}{$\begin{array}{l}\text { Overall sensitivity } \\
\text { Unsubtracted } \\
\text { mixed }\end{array}$} & $\begin{array}{l}\text { Virtual } \\
\text { unenhanced }\end{array}$ & $\begin{array}{c}\text { Unsubtracted } \\
\text { mixed }\end{array}$ & $\begin{array}{c}\text { Virtual } \\
\text { unenhanced }\end{array}$ \\
$86.9 \%$ & $78.2 \%$ & $100 \%$ & $100 \%$ \\
$20 / 23$ stones & $18 / 23$ stones & $18 / 18$ stones & $18 / 18 \%$ \\
\hline
\end{tabular}


Fig. 1 Dual energy (DE) CT urography of a 55-year-old man with macrohaematuria. Coronal CT reconstructions show an inferior calyceal group stone of the left kidney in the true unenhanced series (white arrow in a), characterised as a non-uric acid stone by the dedicated software based on the data of this true unenhanced acquisition (white arrow in $\mathbf{b}$ ). The same calculus is visible on the coronal reconstructions of the mixed nephrographic excretory phase through diluted urine (white arrow in c) and characterised as a non-uric acid calculus based on this image series (white arrow in d)
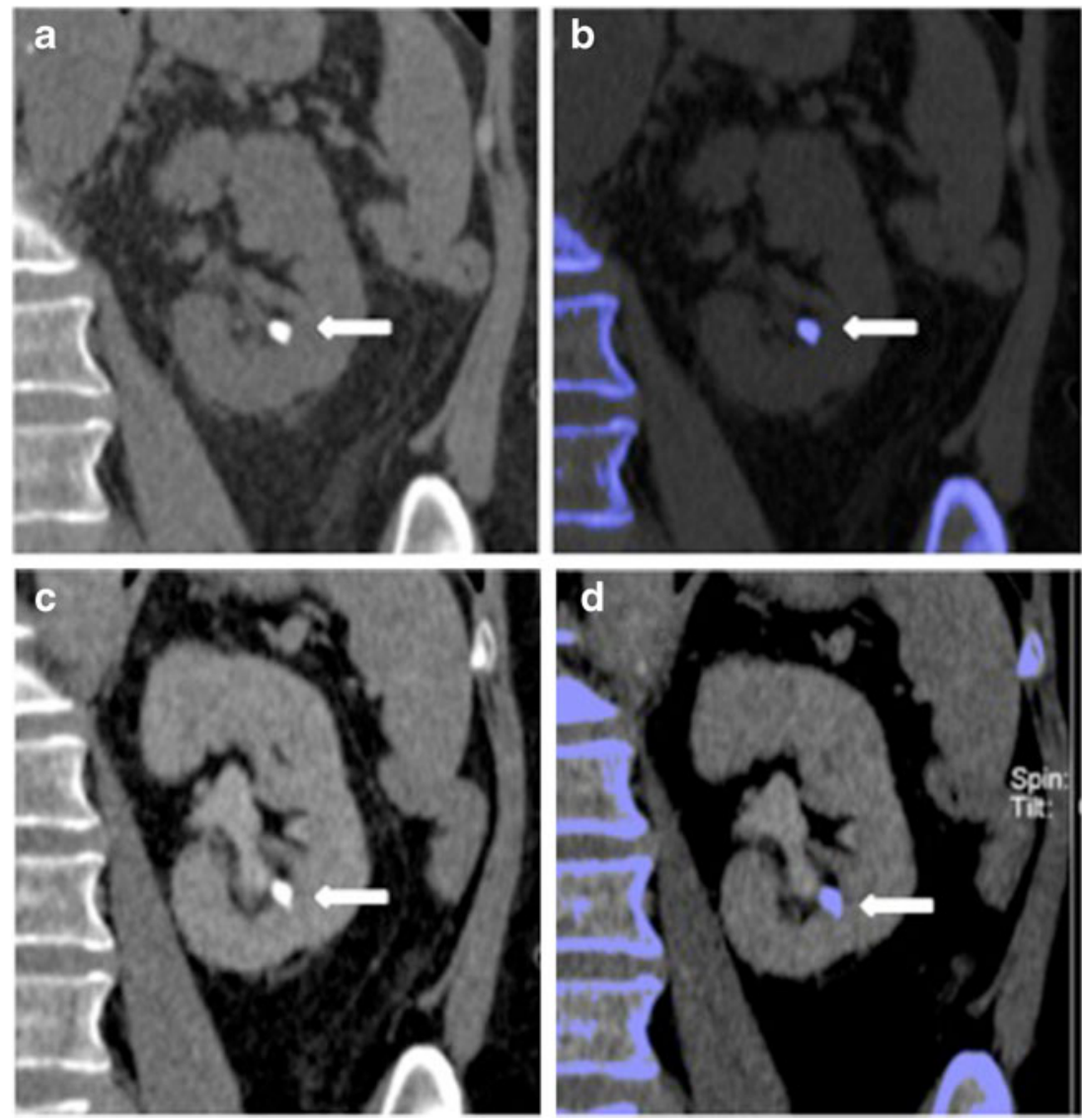

less than $2 \mathrm{~mm}$. The reason for this might be contrast dilution includes a limited number of calculi and further research is in urine due to furosemide injection. Of course, our study needed in the domain.

Fig. 2 DECT urography of a 67year-old man with known bladder cancer. Axial plane CT showing a bladder calculus in the true unenhanced series (white arrow in a), characterised as a uric acid calculus based on the true unenhanced series (white arrow in b). The same calculus shown on the mixed nephrographic excretory phase through diluted urine (white arrow in $\mathbf{c}$ ) and characterised as a uric acid calculus based on the mixed nephrographic excretory phase (white arrow in d). Notice the extensive bladder cancer on the posterior left lateral bladder wall shown on all four figures and the presence of air in the non-

dependent part of the bladder due to cystoscopy immediately before the $\mathrm{CT}$ examination
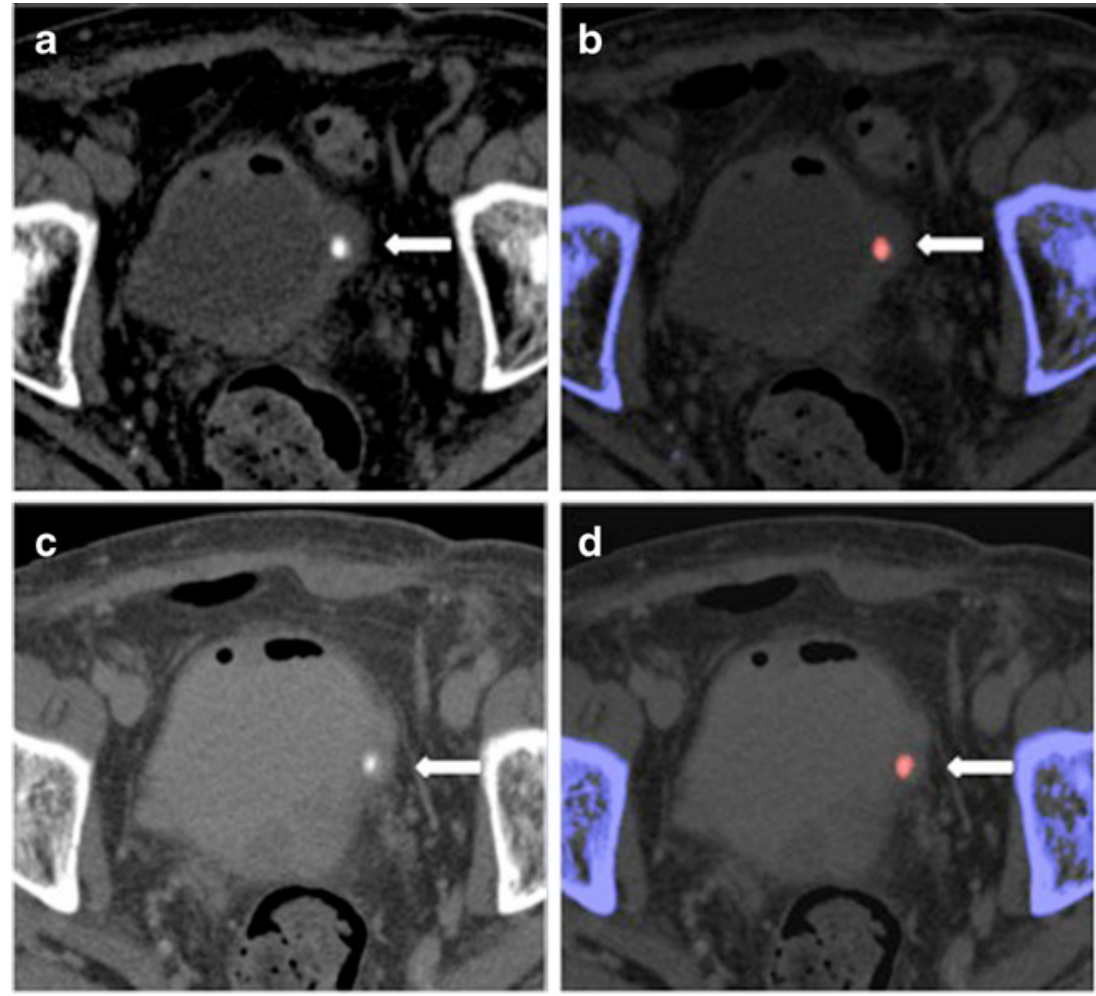
In our study, the overall sensitivity for calculus detection was better for unsubtracted mixed nephrographic excretory phase (86.9 \%, 20 out of 23 calculi) than in the virtual unenhanced series (Table 1). Scheffel et al. [10] found in the same way that virtual unenhanced images created from nephrographic phase CT images had a sensitivity of $74 \%$ (26 out of 35 calculi) while the original unsubtracted nephrographic phase images had a sensitivity of $94 \%$ (33 out of 35 calculi). Thus from both studies it was shown that virtual unenhanced images have no added value in calculus detection compared with the original unsubtracted $\mathrm{CT}$ acquisitions neither in the nephrographic phase alone nor in the mixed nephrographic excretory phase with urine dilution.

Both unsubtracted mixed nephrographic excretory phase and virtual unsubtracted series detected all calculi $\geq 2 \mathrm{~mm}$. It is accepted that up to $98 \%$ of calculi less than $5 \mathrm{~mm}$, especially in the distal ureter may be expected to pass spontaneously [11]. Therefore, according to the guideline of the American Urological Association, observation is the initial treatment of these calculi [11]. However, even small calculi can be the cause of haematuria, but calculi less than $8 \mathrm{~mm}$ are less frequently associated with this condition [12]. Despite these facts, clinicians should be informed concerning the lower sensitivity of enhanced DECT for calculi less than $2 \mathrm{~mm}$. Unenhanced CT has also been shown to have the potential to predict calculus formation, by measuring the density to the tip of the papillae [13] with a mean density of $45.2 \mathrm{vs} 32.1$ $\mathrm{HU}$ for the calculus-forming group of patients versus the normal subjects. Virtual unenhanced series is probably inadequate for the detection of such subtle differences. For these indications as well as for the detection of precalyceal or calyceal calculi less than $2 \mathrm{~mm}$, alternatively, an unenhanced CT limited to the kidneys for the detection of microlithiasis can be proposed for the DECT protocol in the clinical context of haematuria.

In our protocol, urine dilution resulting from furosemide injection prior to the examination could also have potential benefits by facilitating automated calculus size measurements by recently developed algorithms [14] that cannot be applied to conventional pyelographic CT acquisitions where the calculus is surrounded by dense urine. The application of these algorithms to the unsubtracted mixed nephrographic excretory phase or even to the corresponding virtual unenhanced series can be further investigated.

Characterisation of urine calculi is feasible by using the DECT data from unenhanced acquisitions [3-5]. Targeted unenhanced DE acquisitions focused on the calculi, previously identified by low-dose CT, has also been described in the literature [15]. To our knowledge, we show in this study for the first time, in vivo, that calculus characterisation is possible through mildly opacified urine in the mixed nephrographic excretory phase with identical results to the true unenhanced series.

Urine calculi characterisation is of major clinical importance, as the treatment of uric acid calculi consists of chemolysis, by urine alkalinisation. It is a medical treatment, in contrast to shock-wave lithotripsy or surgical treatment that is reserved for non-uric acid calculi $[16,17]$. Currently the evaluation of patients with urinary tract calculi is done by 24 -h urine collection as it allows for calculation of urinary supersaturations for the various salts associated with lithogenesis [17]. This is an indirect technique to estimate stone composition. Unenhanced CT has also been shown to characterise uric acid calculi based on their densities [18]. To our knowledge, the accuracy of the two techniques, DECT and unenhanced single-energy CT, for calculi characterisation has never been compared.

Radiation dose is a major limiting factor for CT urography. Given the multitude of clinical settings and for dose reduction purposes, many different CT urography protocols have been proposed [19] and continue to develop, together with technological progress in the domain of CT. Unenhanced CT is needed mainly for calculi detection and characterisation of renal masses. The nephrographic phase is particularly helpful for appreciation of renal masses, while the excretory phase is essentially used for urothelial tumour detection. Early phase acquisitions, as the socalled "urothelial phase", with an acquisition at $60 \mathrm{~s}$ after contrast injection have also been shown to be of great value for the detection of urothelial tumours by their contrast uptake and have been proposed as a single-phase CT urography protocol for haematuria [20]. Split-bolus iodine contrast injection, with a mixed nephrographic excretory phase, has been widely used for the same purpose, in order to skip at least one acquisition. Triple-bolus CT urography has also been mentioned in the literature, in an effort to minimise radiation dose to the patients [21]. With the advent of DECT, the possibility of reconstructing virtual unenhanced series on the basis of the enhanced CT acquisition offers a potential means to achieve further dose reduction, by skipping the unenhanced acquisition. According to many subsequent clinical studies of dual-source DECT, the radiation doses that the patients are exposed to are similar to those received during conventional single-energy CT [22]. In our hospital we perform most $\mathrm{CT}$ acquisitions with this particular $\mathrm{CT}$ system in DE mode. This way, we can benefit from the potential advantages of DECT as metallic artefacts suppression, or even iodine maps (particularly useful in cases of urothelial or renal tumours, ambiguous renal cysts or adrenal incidental lesions).

Ascenti et al. [23] proposed a single-phase mixed nephrographic excretory phase DECT urography protocol, reporting $85.7 \%$ sensitivity and $98.7 \%$ specificity for urothelial tumours. In this study, the reconstructed virtual unenhanced series used for calculus detection had limited value for calculi less than $2 \mathrm{~mm}$ in diameter. The dose reduction by skipping the unenhanced CT in this study was as high as $45 \%$. In our study, we propose eliminating the unenhanced acquisition, thus lowering the overall radiation dose to the patient by a mean of $9.077 \pm$ $2.917 \mathrm{mSv}$. This corresponds to $32.6 \%$ or $49.3 \%$ of the total dose, depending on which CT urography protocol we chose, according to the patient's risk factors for developing urothelial or renal cancer. 
The major study limitation is the small number of calculi, despite an adequate patient population. This is explained by the fact that indications were more focused on haematuria, urothelial tumours and known or suspected renal tumours. Therefore, any calculi were more of an incidental finding.

In conclusion, our preliminary results show that with a single nephrographic excretory phase with urine dilution, we maintain an excellent sensitivity of $100 \%$ for clinically significant calculi of more than $2 \mathrm{~mm}$ in diameter. At the same time, we can still characterise calculi based on their composition, using the data of the DE acquisition. Thus, the unenhanced CT could be skipped in a DECT urography protocol for patients with haematuria as the enhanced series provides all the necessary information.

\section{References}

1. Cowan NC (2012) CT urography for hematuria. Nat Rev Urol 9:218-226

2. Takahashi N, Hartman RP, Vrtiska TJ et al (2008) Dual-energy CT iodine-subtraction virtual unenhanced technique to detect urinary stones in an iodine-filled collecting system: a phantom study. AJR Am J Roentgenol 190:1169-1173

3. Ascenti G, Siragusa C, Racchiusa S et al (2010) Stone-targeted dualenergy CT: a new diagnostic approach to urinary calculosis. AJR Am J Roentgenol 195:953-958

4. Graser A, Johnson TR, Bader M et al (2008) Dual energy CT characterization of urinary calculi: initial in vitro and clinical experience. Invest Radiol 43:112-119

5. Manglaviti G, Tresoldi S, Guerrer CS et al (2011) In vivo evaluation of the chemical composition of urinary stones using dual-energy CT. AJR Am J Roentgenol 197:W76-W83

6. Wang J, Qu M, Duan X et al (2012) Characterisation of urinary stones in the presence of iodinated contrast medium using dualenergy CT: a phantom study. Eur Radiol 22:2589-2596

7. Takahashi N, Vrtiska TJ, Kawashima A et al (2010) Detectability of urinary stones on virtual nonenhanced images generated at pyelographic-phase dual-energy CT. Radiology 256:184-190

8. Mangold S, Thomas C, Fenchel M et al (2012) Virtual nonenhanced dual-energy CT urography with tin-filter technology: determinants of detection of urinary calculi in the renal collecting system. Radiology 264:119-125
9. Karlo CA, Gnannt R, Winklehner A et al (2013) Split-bolus dual-energy CT urography: protocol optimization and diagnostic performance for the detection of urinary stones. Abdom Imaging 38:1136-1143

10. Scheffel H, Stolzmann P, Frauenfelder T et al (2007) Dual-energy contrast-enhanced computed tomography for the detection of urinary stone disease. Invest Radiol 42:823-829

11. Segura JW, Preminger GM, Assimos DG et al (1997) Ureteral Stones Clinical Guidelines Panel summary report on the management of ureteral calculi. The American Urological Association. J Urol 158: 1915-1921

12. Lallas CD, Liu XS, Chiura AN, Das AK, Bagley DH (2011) Urolithiasis location and size and the association with microhematuria and stone-related symptoms. J Endourol 25:1909-1913

13. Ciudin A, Luque Galvez MP, Salvador Izquierdo R et al (2012) Unenhanced CT findings can predict the development of urinary calculi in stone-free patients. Eur Radiol 22:2050-2056

14. Liden M, Andersson T, Broxvall M, Thunberg P, Geijer H (2012) Urinary stone size estimation: a new segmentation algorithm-based CT method. Eur Radiol 22:731-737

15. Eiber M, Holzapfel K, Frimberger M et al (2012) Targeted dualenergy single-source CT for characterisation of urinary calculi: experimental and clinical experience. Eur Radiol 22:251-258

16. Moran ME, Abrahams HM, Burday DE, Greene TD (2002) Utility of oral dissolution therapy in the management of referred patients with secondarily treated uric acid stones. Urology 59:206-210

17. Xu H, Zisman AL, Coe FL, Worcester EM (2013) Kidney stones: an update on current pharmacological management and future directions. Expert Opin Pharmacother 14:435-447

18. Nakada SY, Hoff DG, Attai S, Heisey D, Blankenbaker D, Pozniak M (2000) Determination of stone composition by noncontrast spiral computed tomography in the clinical setting. Urology 55:816-819

19. Nolte-Ernsting C, Cowan N (2006) Understanding multislice CT urography techniques: Many roads lead to Rome. Eur Radiol 16:2670-2686

20. Metser U, Goldstein MA, Chawla TP, Fleshner NE, Jacks LM, O'Malley ME (2012) Detection of urothelial tumors: comparison of urothelial phase with excretory phase CT urography - a prospective study. Radiology 264:110-118

21. Kekelidze M, Dwarkasing RS, Dijkshoorn ML, Sikorska K, Verhagen PC, Krestin GP (2010) Kidney and urinary tract imaging: triple-bolus multidetector CT urography as a one-stop shop-protocol design, opacification, and image quality analysis. Radiology 255:508-516

22. Kaza RK, Platt JF, Cohan RH, Caoili EM, Al-Hawary MM, Wasnik A (2012) Dual-energy CT with single- and dual-source scanners: current applications in evaluating the genitourinary tract. Radiographics 32:353-369

23. Ascenti G, Mileto A, Gaeta M, Blandino A, Mazziotti S, Scribano E (2013) Single-phase dual-energy CT urography in the evaluation of haematuria. Clin Radiol 68:e87-e94 